\title{
Supplementary information for Deep learning for prediction of the air quality response to emission changes
}

Jia Xing ${ }^{1,2}$, Shuxin Zheng ${ }^{3}$, Dian Ding ${ }^{1,2}$, James T. Kelly ${ }^{4}$, Shuxiao Wang ${ }^{1,2, *}$, Siwei $\mathrm{Li}^{5}$, Tao Qin ${ }^{3, *}$, Mingyuan $\mathrm{Ma}^{6}$, Zhaoxin Dong ${ }^{1,2}$, Carey Jang ${ }^{4}$, Yun Zhu ${ }^{7}$, Haotian Zheng ${ }^{1,2}$, Lu Ren ${ }^{1,2}$, Tie-Yan Liu ${ }^{3}$, Jiming Hao ${ }^{1,2}$

${ }^{1}$ State Key Joint Laboratory of Environmental Simulation and Pollution Control, School of Environment, Tsinghua University, Beijing 100084, China

${ }^{2}$ State Environmental Protection Key Laboratory of Sources and Control of Air Pollution Complex, Beijing 100084, China

${ }^{3}$ Microsoft Research Asia, Beijing 100080, China

${ }^{4}$ Office of Air Quality Planning and Standards, U.S. Environmental Protection Agency, Research Triangle Park, NC 27711, USA

${ }^{5}$ School of Remote Sensing and Information Engineering, Wuhan University, Wuhan 430079, China

${ }^{6}$ School of Electronics Engineering and Computer Science, Peking University, Beijing 100084, China

${ }^{7}$ College of Environment and Energy, South China University of Technology, Guangzhou Higher Education Mega Center, Guangzhou 510006, China

"Corresponding Authors: Shuxiao Wang (email: shxwang@tsinghua.edu.cn; phone: +86-10-62771466; fax: +8610-62773650), Tao Qin (email: taoqin@microsoft.com)

\section{Summary:}

Page S3: Table S1. Control matrix of CMAQ scenarios used for generating the pf-RSM as well as the training and testing dataset for DeepRSM

Page S4: Table S2. Statistics of pf-RSM and DeepRSM performance in predicting the air pollution repsonses

Page S5: Figure S1 Spatial location of four simulation domains

Page S6-7: Figure S2. Spatial and seasonal variation of 14 term coefficients of the response function of $\mathrm{PM}_{2.5}$ (a) and $\mathrm{O}_{3}$ (b) regressed with 40 samples in pf-RSM and predicted by DeepRSM using ambient concentrations (at base and clean conditions) of chemical species related to $\mathrm{PM}_{2.5}$ and $\mathrm{O}_{3}$ (based on CN27 dataset)

Page S8: Figure S3 Statistics of pf-RSM and DeepRSM performance in predicing $\mathrm{O}_{3}$ responses to emissions under different experiment design (an example for Jul 30 under control scenario \#4 in Table $\mathrm{S} 1$, the emission change ratio of $\mathrm{NO}_{\mathrm{x}}, \mathrm{SO}_{2}, \mathrm{NH}_{3}$ and VOC are $-87.5 \%,-12.5 \%,+60.5 \%$, and $-97.2 \%$ )

Page S9: Figure S4 The daily variation of $\mathrm{PM}_{2.5}$ response under two selected scenarios (the \# 18 and 35 in Table S1) 
Page S10: Figure S5 The daily variation of $\mathrm{O}_{3}$ response under two selected scenarios (the \# 18 and 4 in Table S1)

Page S11: Figure S6 The spatial distribution of $\mathrm{PM}_{2.5}$ response under two selected scenarios in $\mathrm{CN} 27$ under -DT experiment design

Page S12: Figure S7 The spatial distribution of $\mathrm{PM}_{2.5}$ response under two selected scenarios in NCP under -DT experiment design

Page S13: Figure S8 The spatial distribution of $\mathrm{PM}_{2.5}$ response under two selected scenarios in FWP under -DT experiment design

Page S14: Figure S9 The spatial distribution of $\mathrm{PM}_{2.5}$ response under two selected scenarios in CYR under -DT experiment design

Page S15: Figure $\mathrm{S} 10$ The spatial distribution of $\mathrm{O}_{3}$ response under two selected scenarios in $\mathrm{CN} 27$ under -DT experiment design

Page S16: Figure $\mathrm{S} 11$ The spatial distribution of $\mathrm{O}_{3}$ response under two selected scenarios in NCP under -DT experiment design

Page S17: Figure $\mathrm{S} 12$ The spatial distribution of $\mathrm{O}_{3}$ response under two selected scenarios in FWP under -DT experiment design

Page S18: Figure $\mathrm{S} 13$ The spatial distribution of $\mathrm{O}_{3}$ response under two selected scenarios in CYR under -DT experiment design

Page S19: Figure S14. The isopleths of $\mathrm{PM}_{2.5}$ response to $\mathrm{NO}_{\mathrm{x}} / \mathrm{VOC}$ (a) and $\mathrm{SO}_{2} / \mathrm{NH}_{3}$ (b) emission change (baseline $=1$ ) predicted by pf-RSM and DeepRSM with -DT for CN27 domain

Page S20: Figure $\mathrm{S} 15$. The isopleths of $\mathrm{O}_{3}$ response to $\mathrm{NO}_{\mathrm{x}} / \mathrm{VOC}$ (a) and $\mathrm{SO}_{2} / \mathrm{NH}_{3}$ (b) emission change (baseline $=1$ ) predicted by pf-RSM and DeepRSM with -DT for CN27 domain 
Table S1. Control matrix of CMAQ scenarios used for generating the pf-RSM as well as the training and testing dataset for DeepRSM

\begin{tabular}{|c|c|c|c|c|}
\hline Cases & $\Delta$ emis- $\mathrm{NO}_{\mathrm{x}}$ & $\Delta$ emis-SO ${ }_{2}$ & $\Delta$ emis- $\mathrm{NH}_{3}$ & $\Delta$ emis-VOC \\
\hline Baseline & 0 & 0 & 0 & 0 \\
\hline Fully-controlled & $-100 \%$ & $-100 \%$ & $-100 \%$ & $-100 \%$ \\
\hline Scenario 1 & $-98.0 \%$ & $-50.0 \%$ & $11.1 \%$ & $28.0 \%$ \\
\hline 2 & $-95.5 \%$ & $50.0 \%$ & $-90.1 \%$ & $-28.0 \%$ \\
\hline 3 & $-92.0 \%$ & $-87.5 \%$ & $58.0 \%$ & $68.0 \%$ \\
\hline 4 & $-87.5 \%$ & $-12.5 \%$ & $60.5 \%$ & $-98.7 \%$ \\
\hline 5 & $-82.0 \%$ & $12.5 \%$ & $-60.5 \%$ & $-53.9 \%$ \\
\hline 6 & $-75.5 \%$ & $87.5 \%$ & $-58.0 \%$ & $54.9 \%$ \\
\hline 7 & $-68.0 \%$ & $-96.9 \%$ & $90.1 \%$ & $-3.7 \%$ \\
\hline 8 & $-59.5 \%$ & $-53.1 \%$ & $-98.9 \%$ & $79.5 \%$ \\
\hline 9 & $-50.0 \%$ & $-21.9 \%$ & $9.7 \%$ & $-94.9 \%$ \\
\hline 10 & $-39.5 \%$ & $71.9 \%$ & $29.8 \%$ & $-37.3 \%$ \\
\hline 11 & $-28.0 \%$ & $-71.9 \%$ & $-82.4 \%$ & $84.3 \%$ \\
\hline 12 & $-15.5 \%$ & $21.9 \%$ & $85.5 \%$ & $18.1 \%$ \\
\hline 13 & $-2.0 \%$ & $53.1 \%$ & $72.6 \%$ & $88.5 \%$ \\
\hline 14 & $12.5 \%$ & $96.9 \%$ & $-46.2 \%$ & $-88.5 \%$ \\
\hline 15 & $28.0 \%$ & $-99.2 \%$ & $-32.8 \%$ & $-18.1 \%$ \\
\hline 16 & $44.5 \%$ & $-75.8 \%$ & $95.6 \%$ & $-84.3 \%$ \\
\hline 17 & $62.0 \%$ & $-36.7 \%$ & $-95.6 \%$ & $37.3 \%$ \\
\hline 18 & $80.5 \%$ & $61.7 \%$ & $32.8 \%$ & $94.9 \%$ \\
\hline 19 & $100.0 \%$ & $-80.5 \%$ & $46.2 \%$ & $-79.5 \%$ \\
\hline 20 & $-80.5 \%$ & $5.5 \%$ & $-72.6 \%$ & $3.7 \%$ \\
\hline 21 & $-62.0 \%$ & $32.0 \%$ & $-85.5 \%$ & $-54.9 \%$ \\
\hline 22 & $-44.5 \%$ & $93.0 \%$ & $82.4 \%$ & $53.9 \%$ \\
\hline 23 & $-28.0 \%$ & $-93.0 \%$ & $-29.8 \%$ & $98.7 \%$ \\
\hline 24 & $-12.5 \%$ & $-32.0 \%$ & $-9.7 \%$ & $-99.9 \%$ \\
\hline 25 & $2.0 \%$ & $-5.5 \%$ & $98.9 \%$ & $-65.4 \%$ \\
\hline 26 & $15.5 \%$ & $80.5 \%$ & $-99.9 \%$ & $33.2 \%$ \\
\hline 27 & $28.0 \%$ & $-61.7 \%$ & $-4.4 \%$ & $-22.9 \%$ \\
\hline 28 & $39.5 \%$ & $36.7 \%$ & $17.6 \%$ & $70.5 \%$ \\
\hline 29 & $50.0 \%$ & $75.8 \%$ & $-87.8 \%$ & $-98.2 \%$ \\
\hline 30 & $59.5 \%$ & $99.2 \%$ & $66.9 \%$ & $-50.8 \%$ \\
\hline 31 & $68.0 \%$ & $-99.8 \%$ & $64.8 \%$ & $60.6 \%$ \\
\hline 32 & $75.5 \%$ & $-87.7 \%$ & $-56.0 \%$ & $0.9 \%$ \\
\hline 33 & $82.0 \%$ & $-43.6 \%$ & $-49.4 \%$ & $81.5 \%$ \\
\hline 34 & $87.5 \%$ & $56.1 \%$ & $92.2 \%$ & $-93.8 \%$ \\
\hline 35 & $92.0 \%$ & $-84.2 \%$ & $-98.0 \%$ & $-33.6 \%$ \\
\hline 36 & $95.5 \%$ & $-3.3 \%$ & $17.2 \%$ & $90.5 \%$ \\
\hline 37 & $98.0 \%$ & $22.1 \%$ & $35.5 \%$ & $22.1 \%$ \\
\hline 38 & $99.5 \%$ & $90.4 \%$ & $-79.4 \%$ & $90.0 \%$ \\
\hline 39 & $100.0 \%$ & $-95.1 \%$ & $95.1 \%$ & $-86.9 \%$ \\
\hline 40 & $-100.0 \%$ & $-42.4 \%$ & $76.1 \%$ & $-13.9 \%$ \\
\hline
\end{tabular}


Table S2. Statistics of pf-RSM and DeepRSM performance in predicting the air pollution repsonses

\begin{tabular}{|c|c|c|c|c|c|c|c|c|c|c|c|c|c|}
\hline $\begin{array}{l}\text { Case } \\
\text { name }\end{array}$ & $\begin{array}{l}\text { Training dat } \\
\text { domain }\end{array}$ & days & Statistics & Jan & Apr & $\begin{array}{l}\mathrm{O}_{3} \\
\mathrm{Jul}\end{array}$ & Oct & Avg & Jan & Apr & $\begin{array}{c}\mathrm{PM}_{2.5} \\
\text { Jul }\end{array}$ & Oct & Avg \\
\hline \multicolumn{14}{|c|}{ pf-RSM (fitting with 20 scenarios $^{2}$ ) } \\
\hline CN27 & CN27 & $26-30$ & MeanNE & 0.03 & 0.01 & 0.01 & 0.02 & 0.02 & 0.02 & 0.02 & 0.03 & 0.02 & 0.03 \\
\hline$-\mathrm{pf}$ & & & 95th MaxNE & 0.09 & 0.03 & 0.02 & 0.06 & 0.05 & 0.10 & 0.12 & 0.14 & 0.11 & 0.12 \\
\hline NCP & $\mathrm{NCP}$ & $26-30$ & MeanNE & 0.02 & 0.01 & 0.01 & 0.05 & 0.02 & 0.05 & 0.04 & 0.06 & 0.07 & 0.06 \\
\hline$-\mathrm{pf}$ & & & 95th MaxNE & 0.08 & 0.03 & 0.03 & 0.18 & 0.08 & 0.22 & 0.19 & 0.33 & 0.29 & 0.26 \\
\hline FWP & FWP & $26-30$ & MeanNE & 0.02 & 0.01 & 0.01 & 0.02 & 0.02 & 0.03 & 0.06 & 0.05 & 0.05 & 0.05 \\
\hline$-p f$ & & & 95th MaxNE & 0.10 & 0.03 & 0.03 & 0.08 & 0.06 & 0.11 & 0.16 & 0.15 & 0.14 & 0.14 \\
\hline CYR & CYR & $26-30$ & MeanNE & 0.01 & 0.01 & 0.00 & 0.01 & 0.01 & 0.02 & 0.05 & 0.05 & 0.04 & 0.04 \\
\hline$-p f$ & & & 95th MaxNE & 0.03 & 0.02 & 0.01 & 0.02 & 0.02 & 0.10 & 0.20 & 0.19 & 0.16 & 0.16 \\
\hline \multicolumn{14}{|c|}{ Temporal transfer (only use the self dataset) } \\
\hline CN27 & CN27 & $1-25$ & MeanNE & 0.01 & 0.01 & 0.01 & 0.01 & 0.01 & 0.02 & 0.02 & 0.03 & 0.02 & 0.02 \\
\hline -TT & & & 95th MaxNE & 0.05 & 0.03 & 0.02 & 0.04 & 0.04 & 0.07 & 0.09 & 0.11 & 0.08 & 0.09 \\
\hline NCP & $\mathrm{NCP}$ & $1-25$ & MeanNE & 0.01 & 0.01 & 0.01 & 0.02 & 0.01 & 0.01 & 0.01 & 0.01 & 0.05 & 0.02 \\
\hline -TT & & & 95th MaxNE & 0.08 & 0.03 & 0.04 & 0.10 & 0.06 & 0.06 & 0.05 & 0.06 & 0.23 & 0.10 \\
\hline FWP & FWP & $1-25$ & MeanNE & 0.02 & 0.01 & 0.01 & 0.02 & 0.01 & 0.03 & 0.04 & 0.04 & 0.04 & 0.04 \\
\hline$-\mathrm{TT}$ & & & 95th MaxNE & 0.07 & 0.03 & 0.05 & 0.06 & 0.05 & 0.09 & 0.11 & 0.12 & 0.11 & 0.11 \\
\hline CYR & CYR & $1-25$ & MeanNE & 0.01 & 0.01 & 0.01 & 0.01 & 0.01 & 0.02 & 0.04 & 0.04 & 0.03 & 0.03 \\
\hline -TT & & & 95th MaxNE & 0.03 & 0.02 & 0.02 & 0.04 & 0.03 & 0.07 & 0.13 & 0.13 & 0.11 & 0.11 \\
\hline \multicolumn{14}{|c|}{ Domain transfer (only use other dataset) } \\
\hline CN27 & NCP/FWP/CYR & $1-30$ & MeanNE & 0.02 & 0.01 & 0.01 & 0.02 & 0.01 & 0.03 & 0.03 & 0.03 & 0.03 & 0.03 \\
\hline -DT & & & 95th MaxNE & 0.09 & 0.05 & 0.03 & 0.08 & 0.06 & 0.11 & 0.10 & 0.13 & 0.10 & 0.11 \\
\hline NCP & CN27/FWP/CYR & $1-30$ & MeanNE & 0.02 & 0.01 & 0.01 & 0.03 & 0.02 & 0.02 & 0.02 & 0.01 & 0.06 & 0.03 \\
\hline -DT & & & 95th MaxNE & 0.09 & 0.05 & 0.04 & 0.11 & 0.07 & 0.09 & 0.07 & 0.06 & 0.23 & 0.11 \\
\hline FWP & $\mathrm{CN} 27 / \mathrm{NCP} / \mathrm{CYR}$ & $1-30$ & MeanNE & 0.02 & 0.01 & 0.01 & 0.01 & 0.02 & 0.03 & 0.04 & 0.04 & 0.04 & 0.04 \\
\hline -DT & & & 95th MaxNE & 0.09 & 0.03 & 0.05 & 0.07 & 0.06 & 0.09 & 0.11 & 0.11 & 0.11 & 0.10 \\
\hline CYR & $\mathrm{CN} 27 / \mathrm{NCP} / \mathrm{FWP}$ & $1-30$ & MeanNE & 0.02 & 0.01 & 0.01 & 0.01 & 0.01 & 0.02 & 0.04 & 0.04 & 0.02 & 0.03 \\
\hline -DT & & & 95th MaxNE & 0.12 & 0.04 & 0.02 & 0.05 & 0.06 & 0.09 & 0.13 & 0.13 & 0.11 & 0.12 \\
\hline \multicolumn{14}{|c|}{ Finetune 5 (use other dataset combined with 5 days of self-dataset) } \\
\hline $\mathrm{CN} 27$ & NCP/FWP/CYR & $1-30$ & MeanNE & 0.02 & 0.01 & 0.01 & 0.01 & 0.01 & 0.02 & 0.02 & 0.02 & 0.02 & 0.02 \\
\hline -DTF5 & $+\mathrm{CN} 27$ & $1-5$ & 95th MaxNE & 0.06 & 0.03 & 0.02 & 0.04 & 0.04 & 0.08 & 0.09 & 0.10 & 0.08 & 0.09 \\
\hline NCP & CN27/FWP/CYR & $1-30$ & MeanNE & 0.02 & 0.01 & 0.01 & 0.02 & 0.01 & 0.01 & 0.01 & 0.01 & 0.05 & 0.02 \\
\hline -DTF5 & $+\mathrm{NCP}$ & $1-5$ & 95th MaxNE & 0.08 & 0.04 & 0.04 & 0.10 & 0.07 & 0.07 & 0.06 & 0.06 & 0.22 & 0.10 \\
\hline FWP & $\mathrm{CN} 27 / \mathrm{NCP} / \mathrm{CYR}$ & $1-30$ & MeanNE & 0.02 & 0.01 & 0.01 & 0.02 & 0.01 & 0.03 & 0.04 & 0.04 & 0.04 & 0.04 \\
\hline -DTF5 & +FWP & $1-5$ & 95th MaxNE & 0.08 & 0.03 & 0.05 & 0.06 & 0.06 & 0.09 & 0.11 & 0.11 & 0.11 & 0.10 \\
\hline CYR & $\mathrm{CN} 27 / \mathrm{NCP} / \mathrm{FWP}$ & $1-30$ & MeanNE & 0.01 & 0.01 & 0.01 & 0.01 & 0.01 & 0.02 & 0.03 & 0.03 & 0.03 & 0.03 \\
\hline -DTF5 & $+\mathrm{CYR}$ & $1-5$ & 95th MaxNE & 0.04 & 0.03 & 0.02 & 0.04 & 0.03 & 0.07 & 0.13 & 0.11 & 0.10 & 0.10 \\
\hline \multicolumn{14}{|c|}{ Finetune 20 (use other dataset combined with 20 days of self-dataset) } \\
\hline CN27 & $\mathrm{NCP} / \mathrm{FWP} / \mathrm{CYR}$ & $1-30$ & MeanNE & 0.01 & 0.01 & 0.01 & 0.01 & 0.01 & 0.02 & 0.02 & 0.02 & 0.02 & 0.02 \\
\hline -DTF20 & $+\mathrm{CN} 27$ & $1-20$ & 95th MaxNE & 0.06 & 0.03 & 0.02 & 0.04 & 0.04 & 0.07 & 0.08 & 0.10 & 0.08 & 0.08 \\
\hline $\mathrm{NCP}$ & CN27/FWP/CYR & $1-30$ & MeanNE & 0.01 & 0.01 & 0.01 & 0.02 & 0.01 & 0.01 & 0.01 & 0.01 & 0.05 & 0.02 \\
\hline -DTF20 & $+\mathrm{NCP}$ & $1-20$ & 95th MaxNE & 0.08 & 0.03 & 0.04 & 0.10 & 0.06 & 0.06 & 0.05 & 0.05 & 0.23 & 0.10 \\
\hline FWP & $\mathrm{CN} 27 / \mathrm{NCP} / \mathrm{CYR}$ & $1-30$ & MeanNE & 0.02 & 0.01 & 0.01 & 0.02 & 0.01 & 0.03 & 0.04 & 0.04 & 0.04 & 0.03 \\
\hline -DTF20 & + FWP & $1-20$ & 95th MaxNE & 0.07 & 0.03 & 0.05 & 0.06 & 0.05 & 0.08 & 0.11 & 0.11 & 0.11 & 0.10 \\
\hline CYR & $\mathrm{CN} 27 / \mathrm{NCP} / \mathrm{FWP}$ & $1-30$ & MeanNE & 0.01 & 0.01 & 0.01 & 0.01 & 0.01 & 0.02 & 0.03 & 0.03 & 0.03 & 0.03 \\
\hline -DTF20 & + CYR & $1-20$ & 95th MaxNE & 0.04 & 0.02 & 0.02 & 0.03 & 0.03 & 0.06 & 0.12 & 0.10 & 0.10 & 0.10 \\
\hline \multicolumn{14}{|c|}{ Finetune 20 with optimized polynomial structure (use other dataset combined with 20 days of self-dataset) } \\
\hline CN27 & NCP/FWP/CYR & $1-30$ & MeanNE & 0.01 & 0.01 & 0.01 & 0.01 & 0.01 & 0.02 & 0.02 & 0.02 & 0.02 & 0.02 \\
\hline -PolyDTF20 & $+\mathrm{CN} 27$ & $1-20$ & 95th MaxNE & 0.05 & 0.03 & 0.02 & 0.04 & 0.03 & 0.06 & 0.07 & 0.09 & 0.07 & 0.07 \\
\hline $\mathrm{NCP}$ & CN27/FWP/CYR & $1-30$ & MeanNE & 0.01 & 0.01 & 0.01 & 0.02 & 0.01 & 0.01 & 0.01 & 0.01 & 0.05 & 0.02 \\
\hline -PolyDTF20 & $+\mathrm{NCP}$ & $1-20$ & 95th MaxNE & 0.07 & 0.03 & 0.04 & 0.10 & 0.06 & 0.05 & 0.05 & 0.05 & 0.23 & 0.09 \\
\hline FWP & $\mathrm{CN} 27 / \mathrm{NCP} / \mathrm{CYR}$ & $1-30$ & MeanNE & 0.02 & 0.01 & 0.01 & 0.01 & 0.01 & 0.02 & 0.03 & 0.03 & 0.03 & 0.03 \\
\hline -PolyDTF20 & + FWP & $1-20$ & 95th MaxNE & 0.06 & 0.03 & 0.04 & 0.06 & 0.05 & 0.07 & 0.08 & 0.09 & 0.09 & 0.08 \\
\hline CYR & $\mathrm{CN} 27 / \mathrm{NCP} / \mathrm{FWP}$ & $1-30$ & MeanNE & 0.01 & 0.01 & 0.01 & 0.01 & 0.01 & 0.02 & 0.02 & 0.03 & 0.02 & 0.02 \\
\hline -PolyDTF20 & $+\mathrm{CYR}$ & $1-20$ & 95th MaxNE & 0.03 & 0.02 & 0.02 & 0.03 & 0.03 & 0.06 & 0.08 & 0.10 & 0.07 & 0.08 \\
\hline
\end{tabular}

${ }^{1}$ the same test set is applied for all experiments, i.e., the last five days (26-30) in each domain

2 the 40 scenarios were split into two parts, i.e., a 20 used for fitting pf-RSM and the rest used for testing, then averaging both 
Terrain elevation (unit m)

CN27 (China):

- $182 \times 232$ grids ( $27 \mathrm{~km}$ resolution) NCP (North China Plain):

- $162 \times 198$ grids (9km resolution) FWP (Fen Wei Plain):

- $126 \times 147$ grids (9km resolution) CYR (Chuan Yu Region):

- $138 \times 168$ grids (9km resolution)
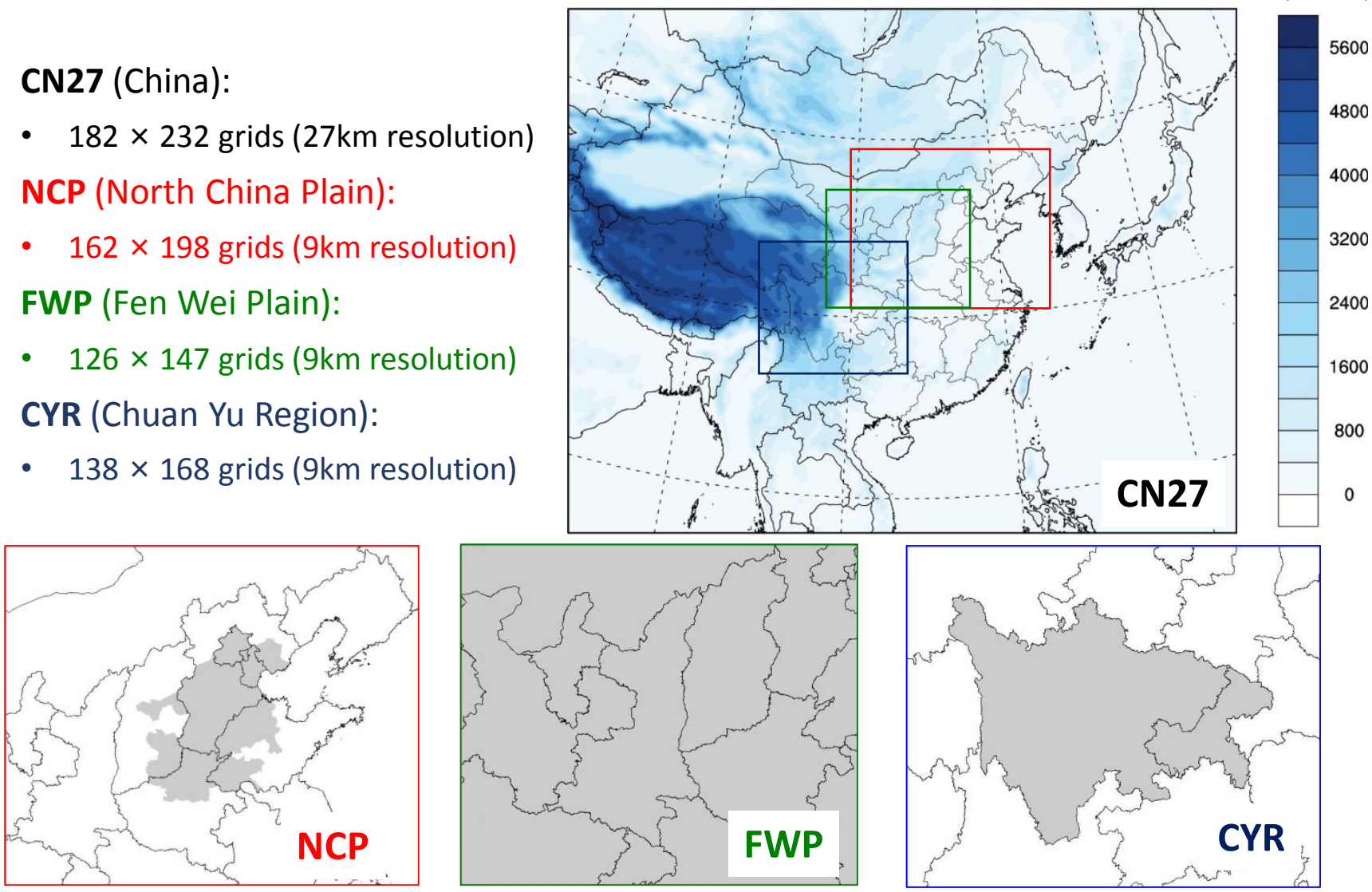

Figure S1 Spatial location of four simulation domains (the shadow area in NCP, FWP and CYR represents the target region to be controlled) 


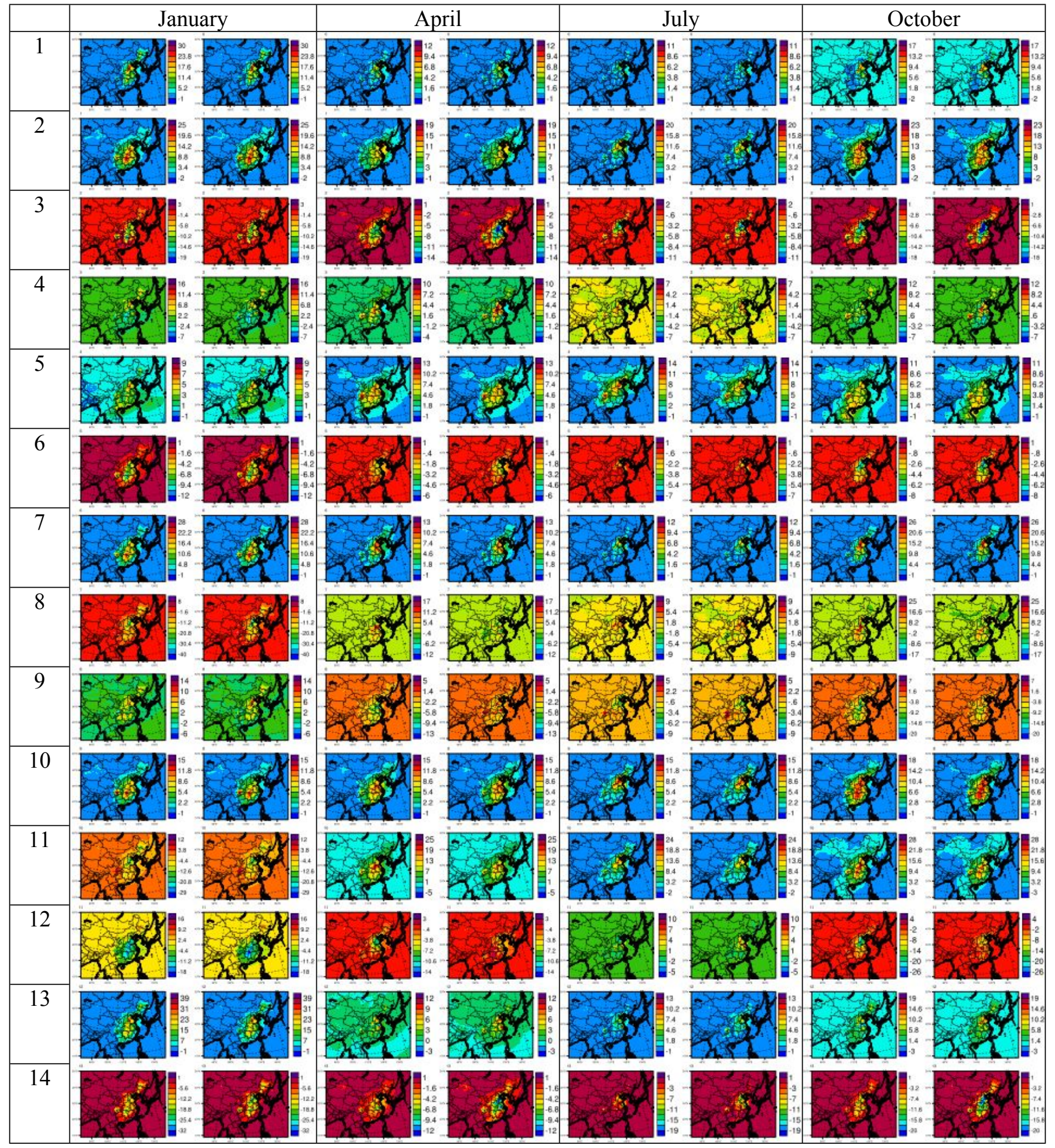

(a) 


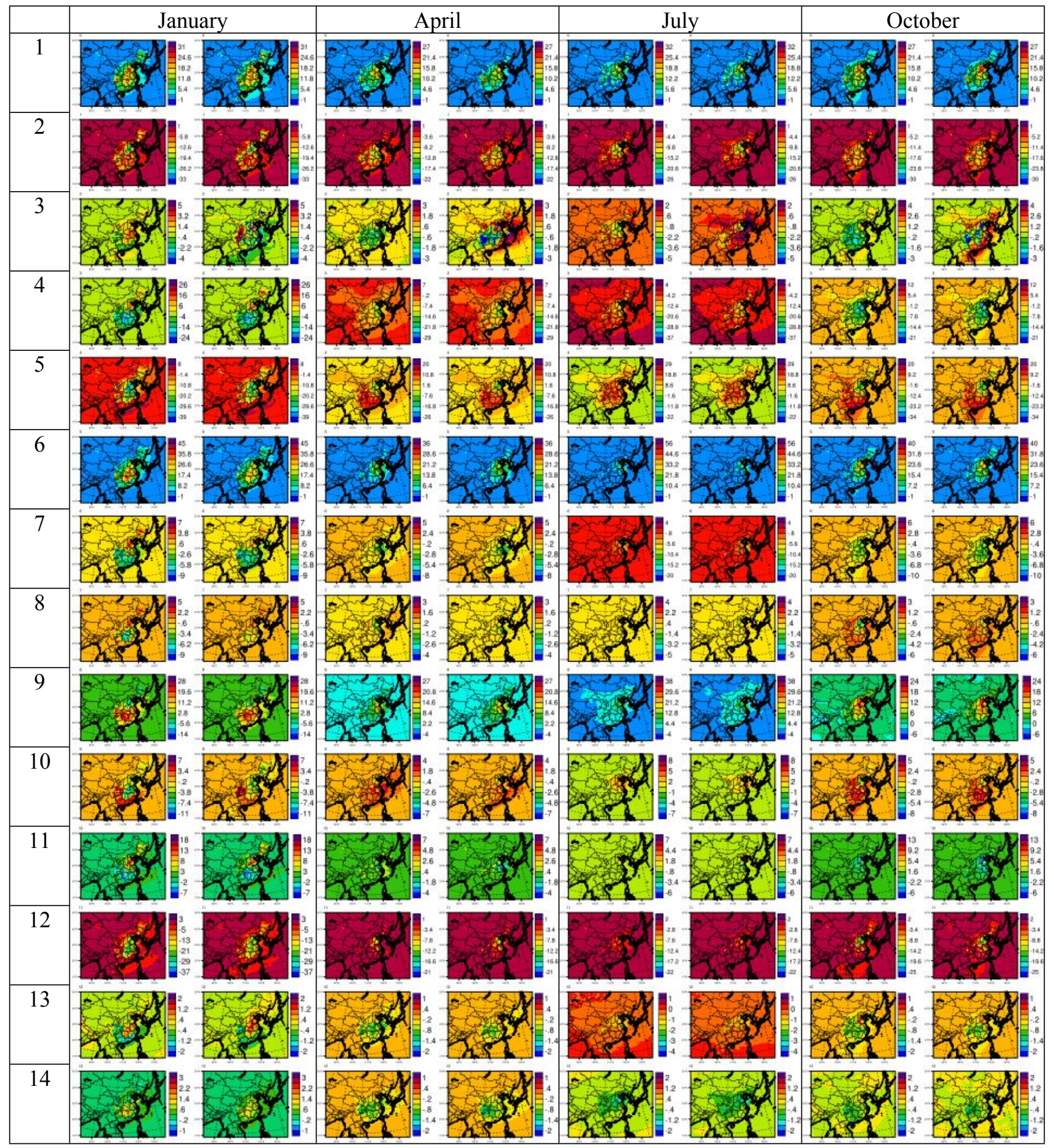

(b)

Figure S2. Spatial and seasonal variation of 14 term coefficients of the response function of $\mathrm{PM}_{2.5}$ (a) and $\mathrm{O}_{3}$ (b) regressed with 40 samples in pf-RSM and predicted by DeepRSM using ambient concentrations (at base and clean conditions) of chemical species related to $\mathrm{PM}_{2.5}$ and $\mathrm{O}_{3}$ (based on CN27 dataset) 

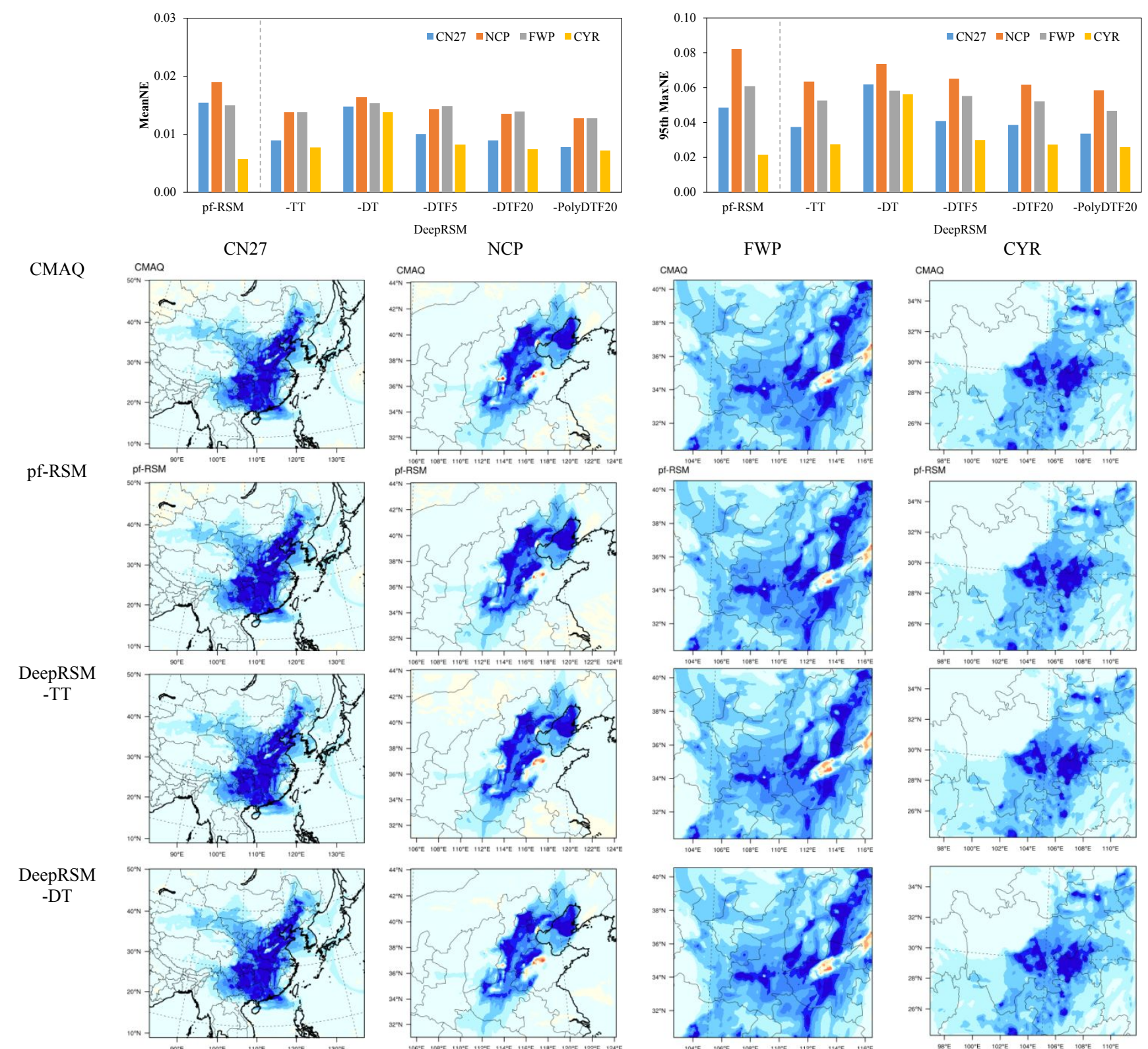

DeepRSM

PolyDTF20
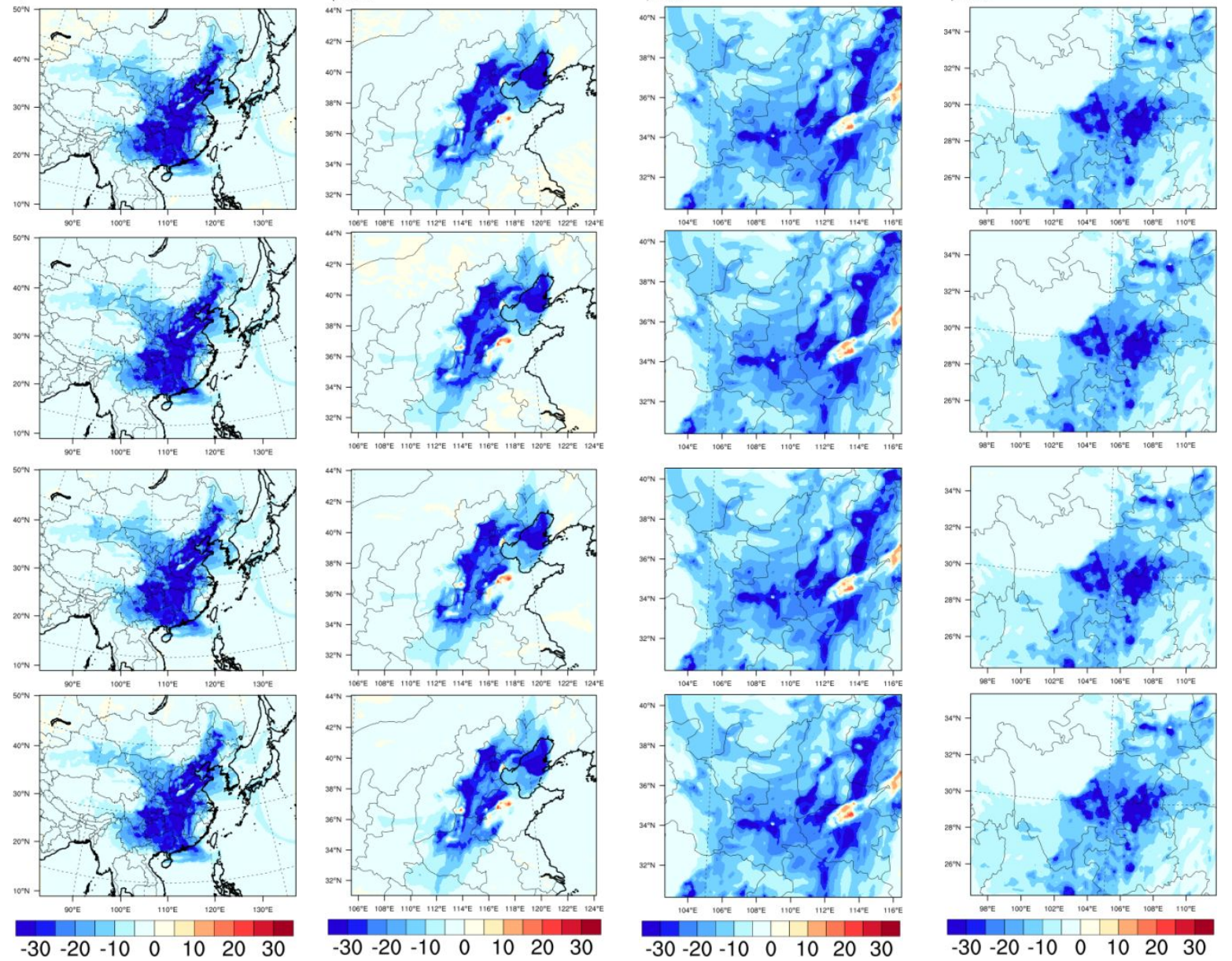

Figure S3 Statistics of pf-RSM and DeepRSM performance in predicing $\mathrm{O}_{3}$ responses to emissions under different experiment design (an example for Jul 30 under control scenario \#4 in Table S1, the emission change ratio of $\mathrm{NO}_{\mathrm{x}}, \mathrm{SO}_{2}, \mathrm{NH}_{3}$ and $\mathrm{VOC}$ are $-87.5 \%,-12.5 \%,+60.5 \%$, and $-97.2 \%$ ) 

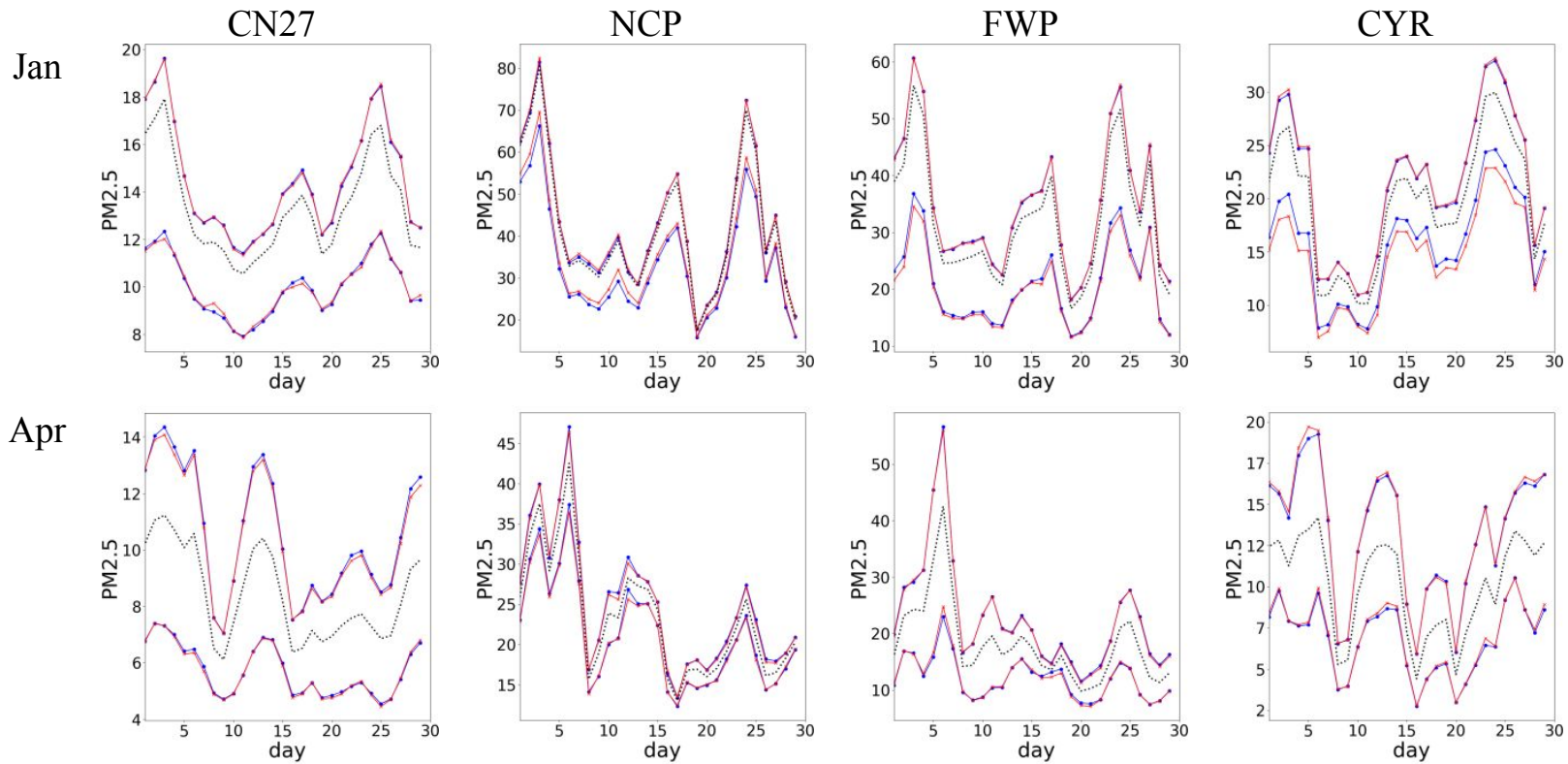

Jul
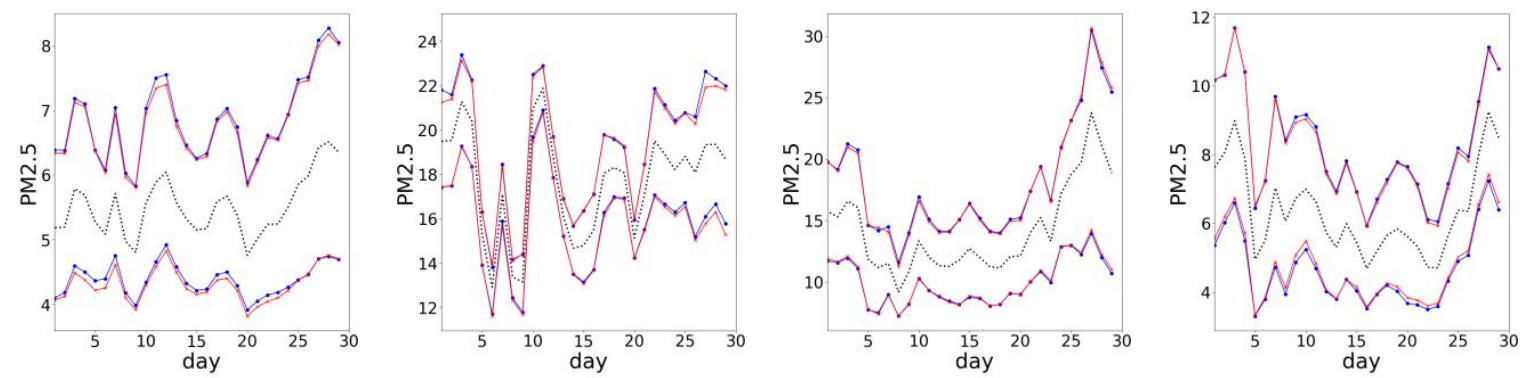

Oct
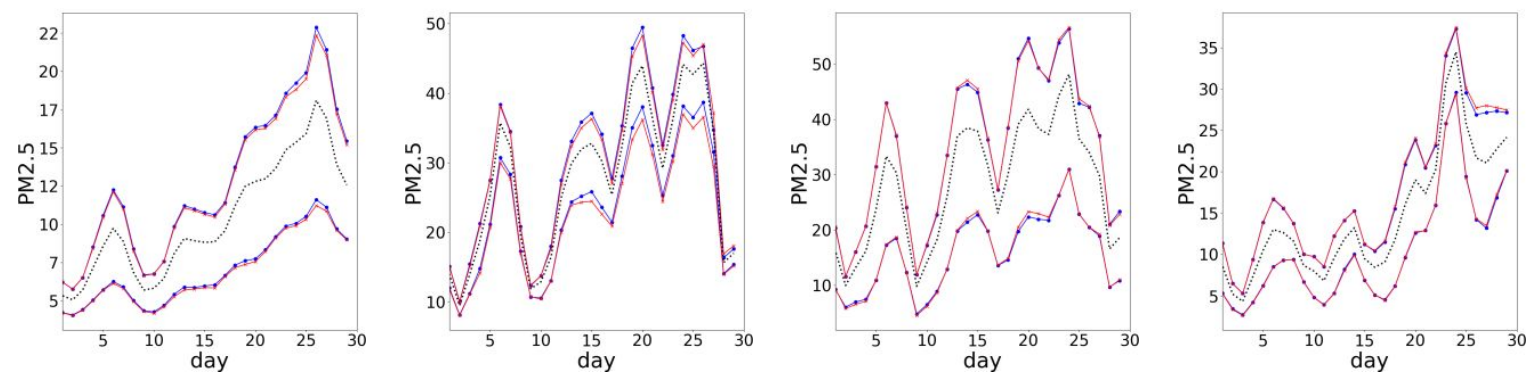

Figure S4 The daily variation of $\mathrm{PM}_{2.5}$ response under two selected scenarios (the \# 18 and 35 in Table

$\mathrm{S} 1$, the emission change ratio of $\mathrm{NO}_{\mathrm{x}}, \mathrm{SO}_{2}, \mathrm{NH}_{3}$ and $\mathrm{VOC}$ are $80.5 \%, 61.7 \%, 32.8 \%$, and $94.9 \%$ respectivley in \# 18 shown as upper lines, and 92.0\%, -84.2\%, -98.0\%, and $-33.6 \%$ in \#35 shown as lower lines; black dash lines represent the baseline, blue lines represnt CMAQ simulation, red lines represent DeepRSM prediction under -DT experiment design) 

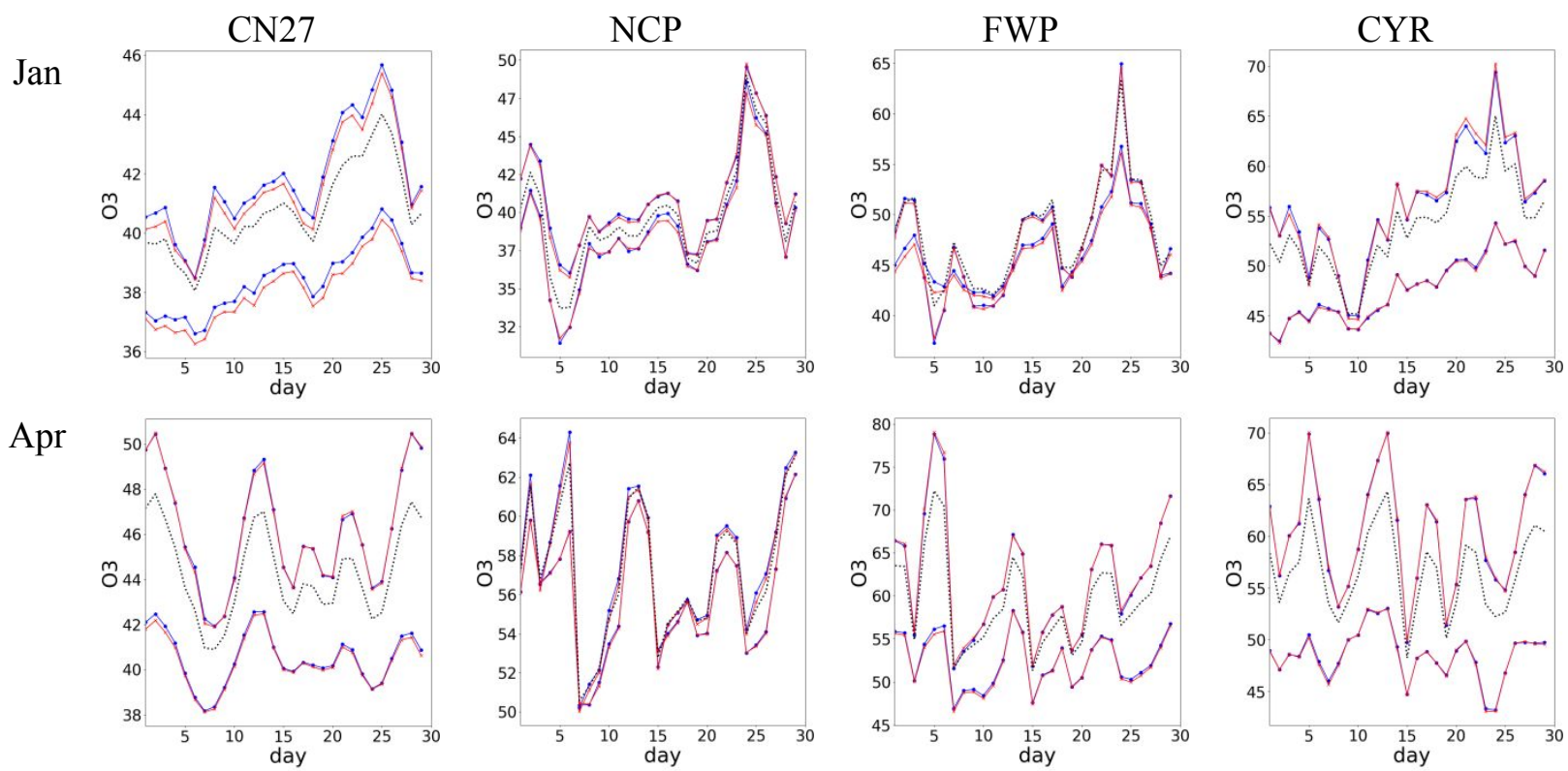

Jul
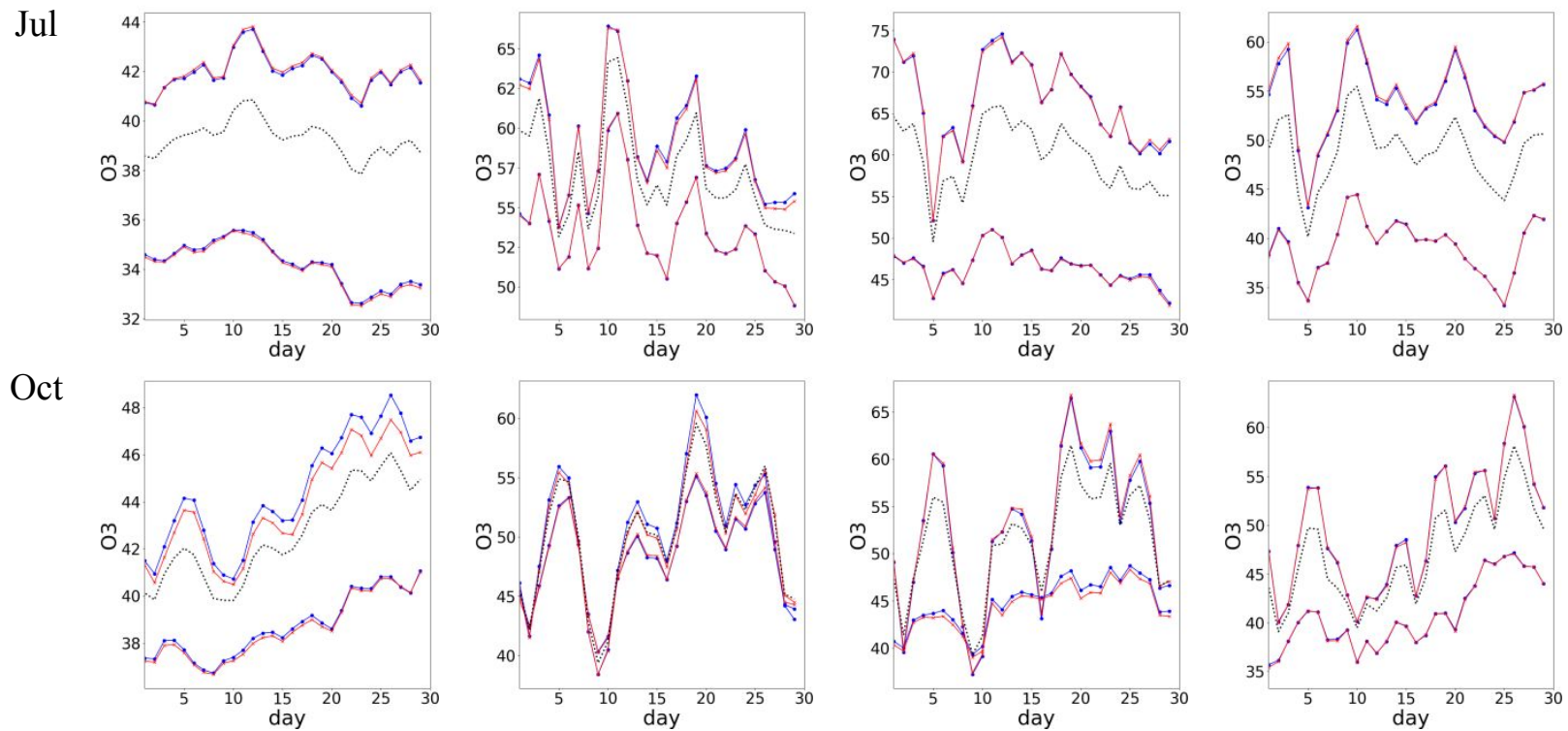

Figure $\mathrm{S} 5$ The daily variation of $\mathrm{O}_{3}$ response under two selected scenarios (the \# 18 and 4 in Table $\mathrm{S} 1$,

the emission change ratio of $\mathrm{NO}_{\mathrm{x}}, \mathrm{SO}_{2}, \mathrm{NH}_{3}$ and $\mathrm{VOC}$ are $80.5 \%, 61.7 \%, 32.8 \%$, and $94.9 \%$ respectivley in \# 18 shown as upper lines, and $-87.5 \%,-12.5 \%,+60.5 \%$, and $-97.2 \%$ in \#4 shown as lower lines; black dash lines represent the baseline, blue lines represnt CMAQ simulation, red lines represent DeepRSM prediction under -DT experiment design) 

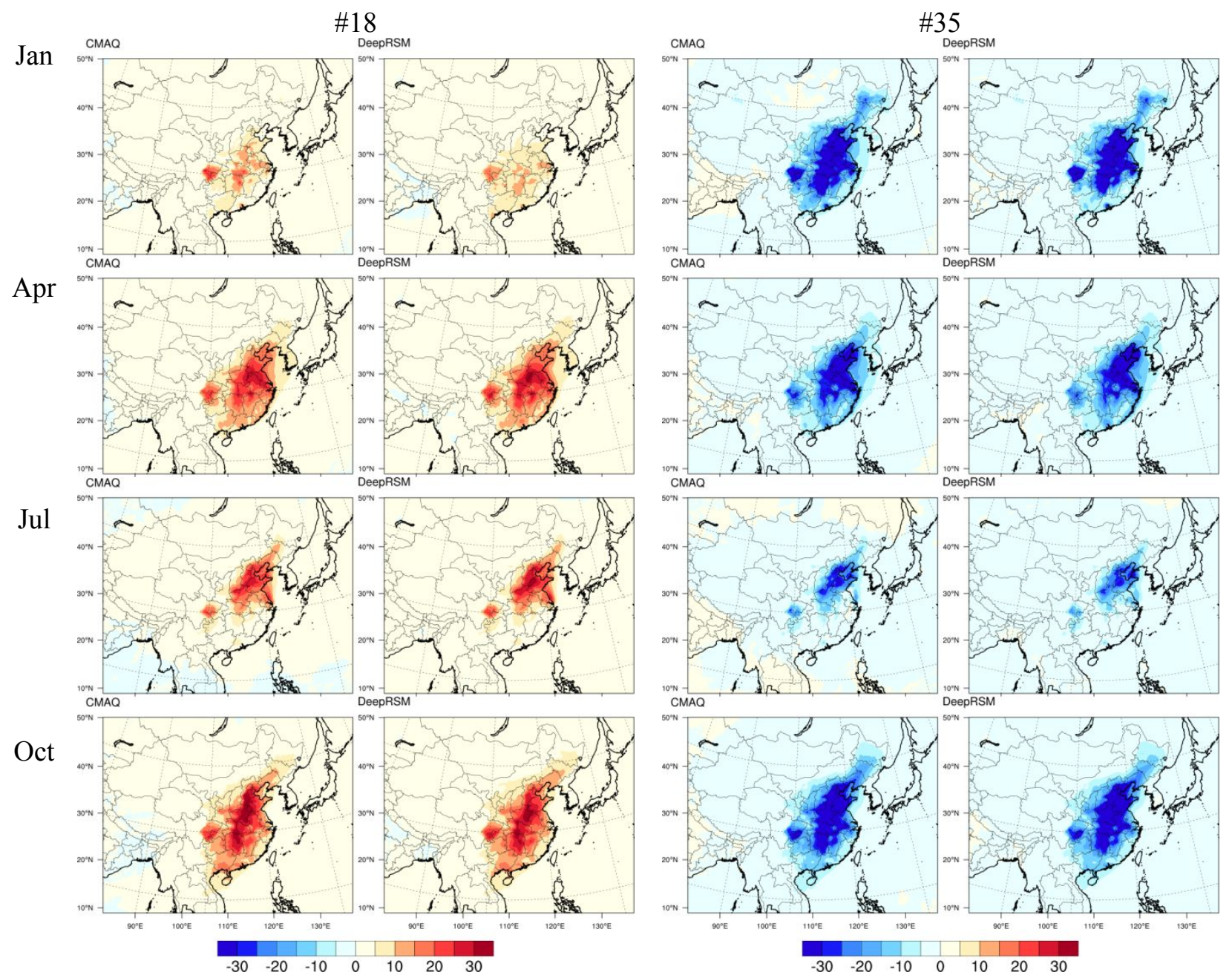

Figure S6 The spatial distribution of $\mathrm{PM}_{2.5}$ response under two selected scenarios in $\mathrm{CN} 27$ under -DT experiment design (the \# 18 and 35 in Table $\mathrm{S} 1$, the emission change ratio of $\mathrm{NO}_{\mathrm{x}}, \mathrm{SO}_{2}, \mathrm{NH}_{3}$ and $\mathrm{VOC}$ are $80.5 \%, 61.7 \%, 32.8 \%$, and $94.9 \%$ respectivley in $\# 18$, and $92.0 \%,-84.2 \%,-98.0 \%$, and $-33.6 \%$ in $\# 35$, monthly averages) 

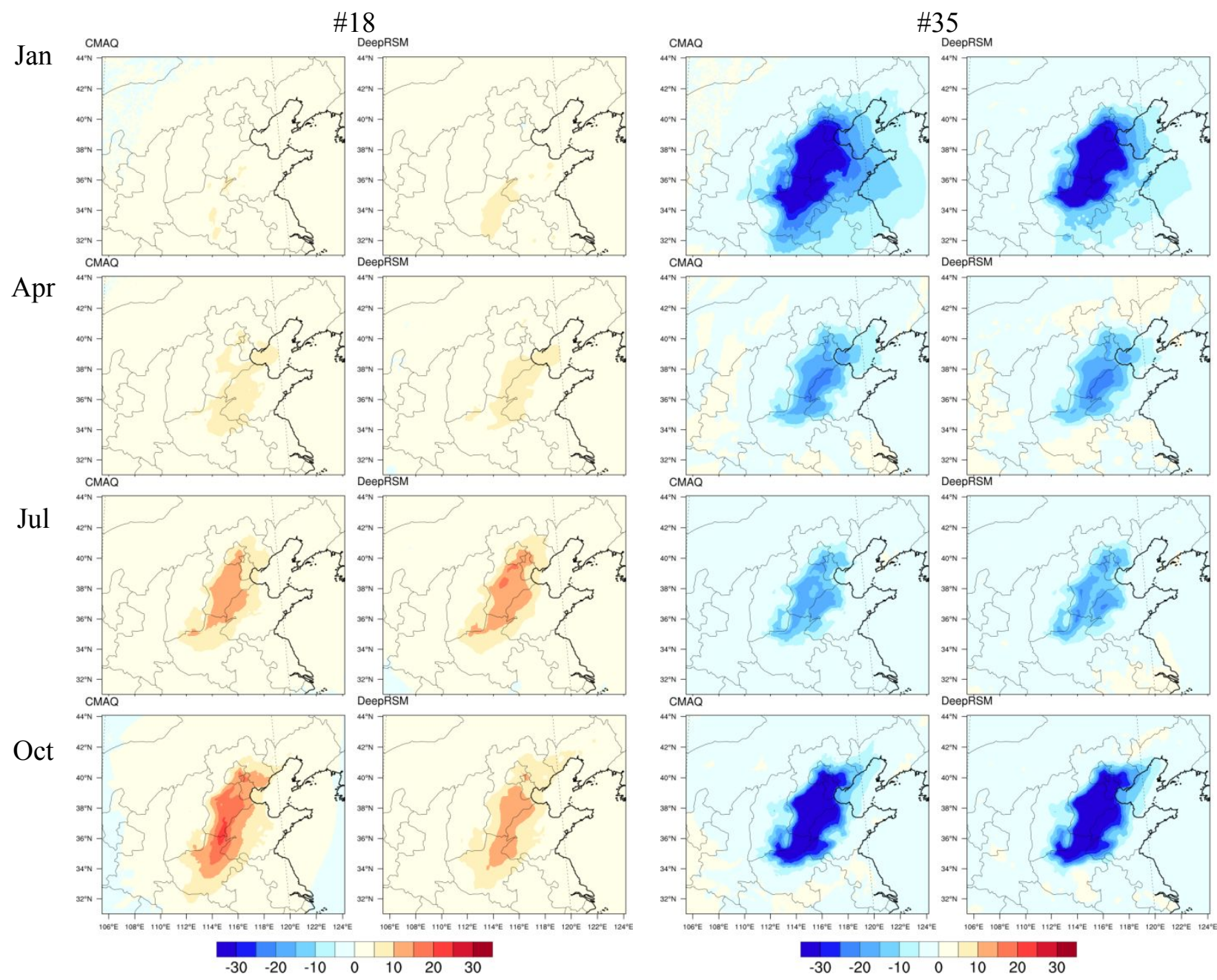

Figure S7 same as Figure S6 but for NCP 

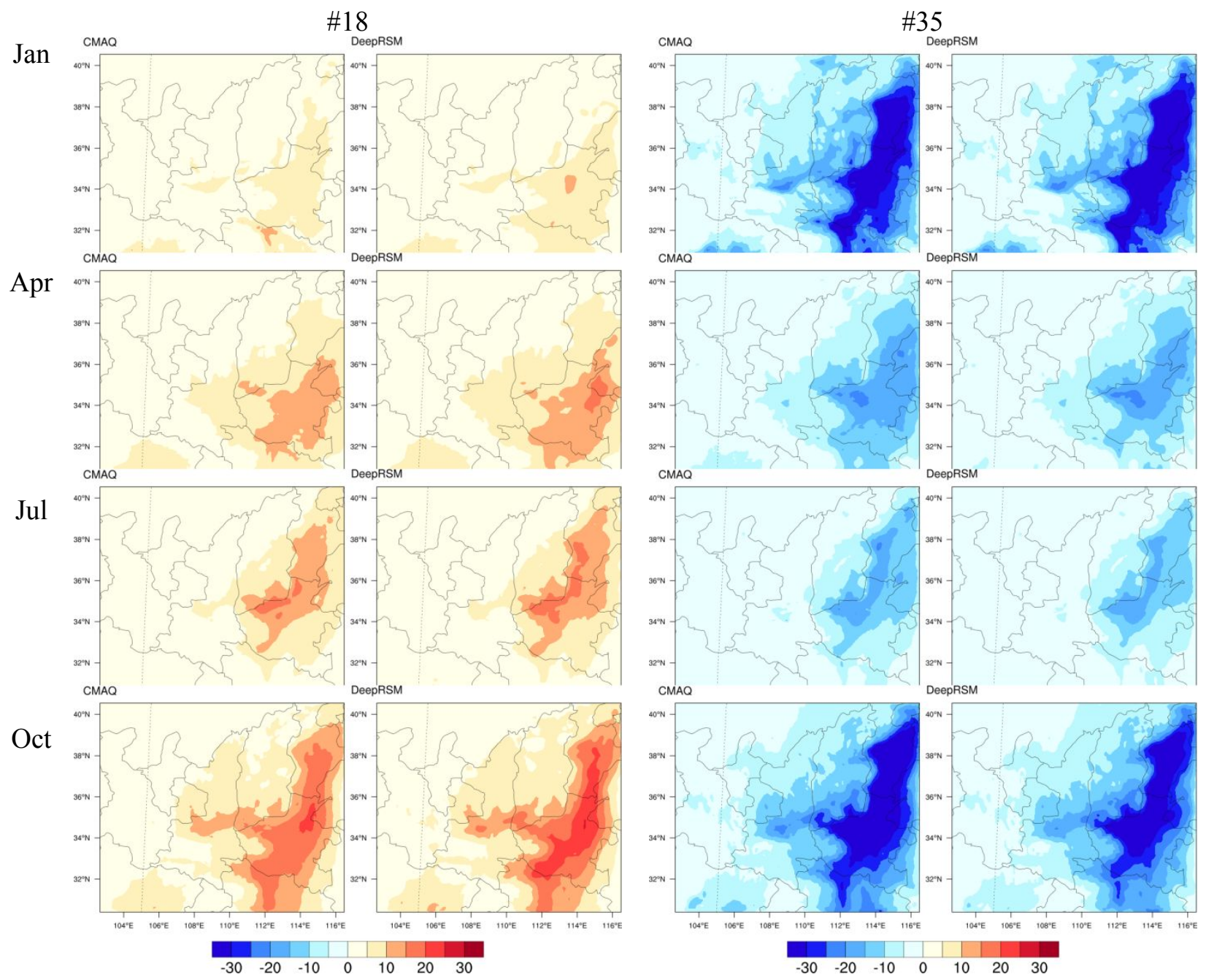

Figure S8 same as Figure S6 but for FWP 

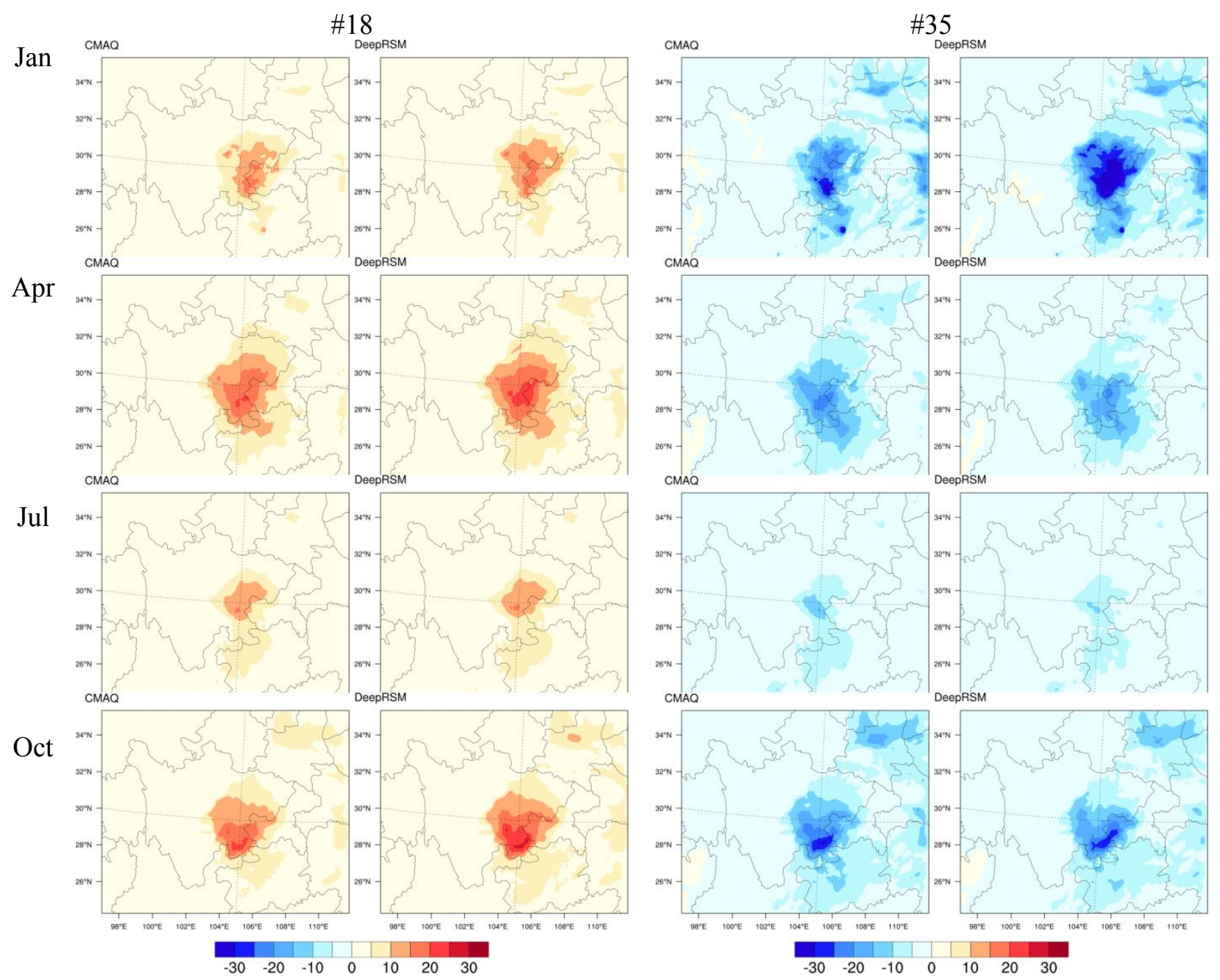

Figure S9 same as Figure S6 but for CYR 

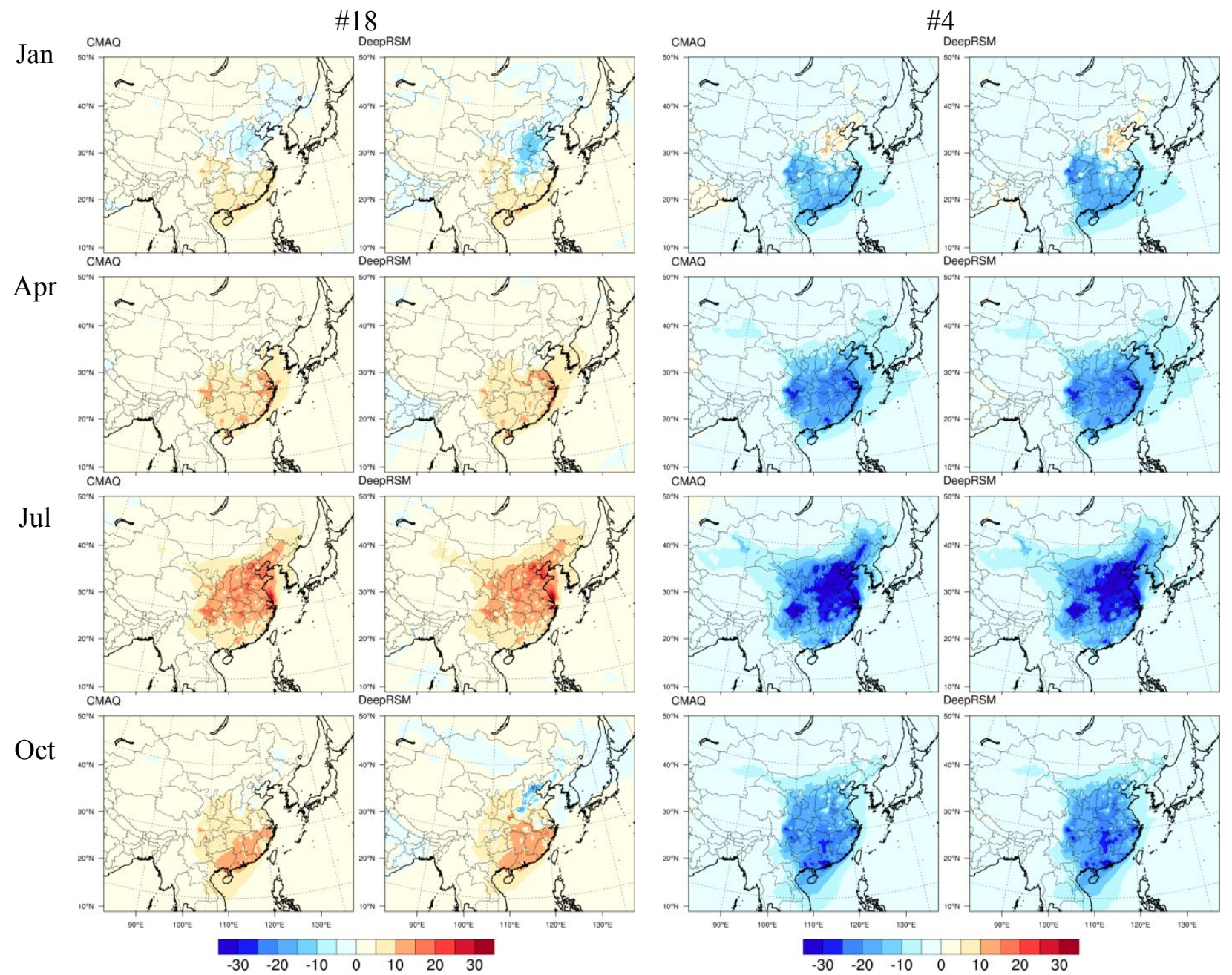

Figure $\mathrm{S} 10$ The spatial distribution of $\mathrm{O}_{3}$ response under two selected scenarios in CN27 under -DT experiment design (the \# 18 and 4 in Table $\mathrm{S} 1$, the emission change ratio of $\mathrm{NO}_{\mathrm{x}}, \mathrm{SO}_{2}, \mathrm{NH}_{3}$ and $\mathrm{VOC}$ are $80.5 \%, 61.7 \%, 32.8 \%$, and $94.9 \%$ respectivley in $\# 18$, and $-87.5 \%,-12.5 \%,+60.5 \%$, and $-97.2 \%$ in $\# 4$, monthly averages) 

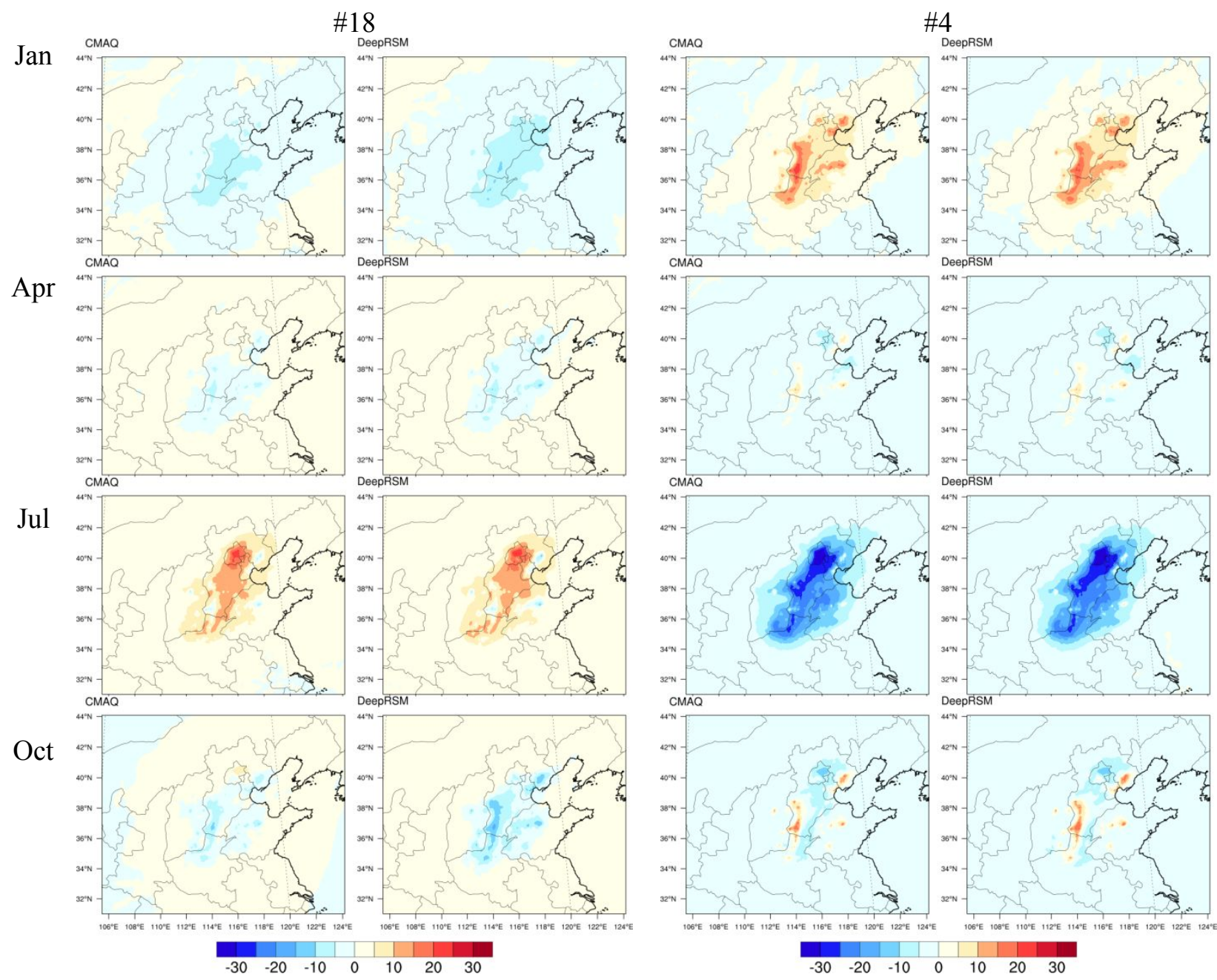

Figure S11 same as Figure S10 but for NCP 

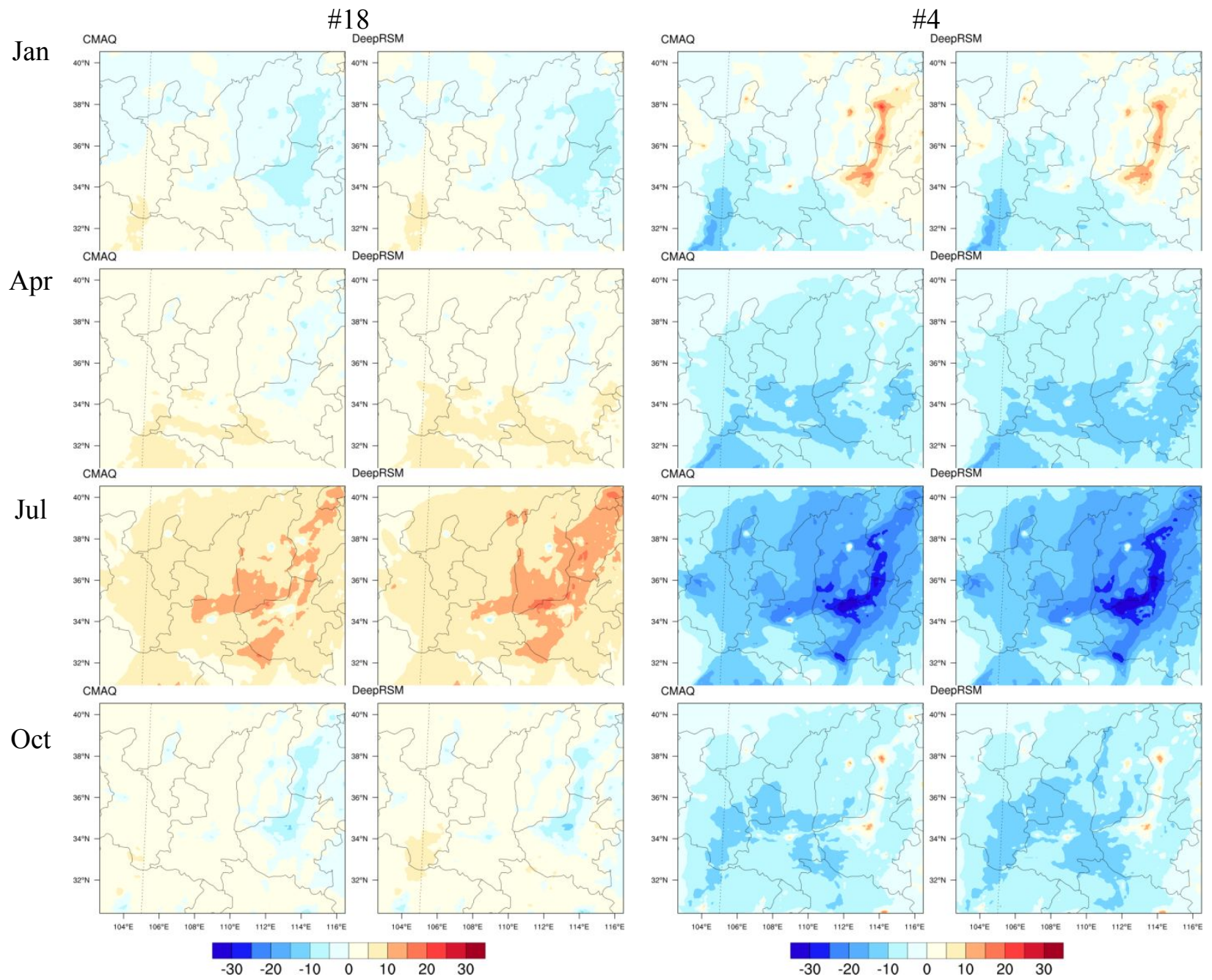

Figure S12 same as Figure S10 but for FWP 

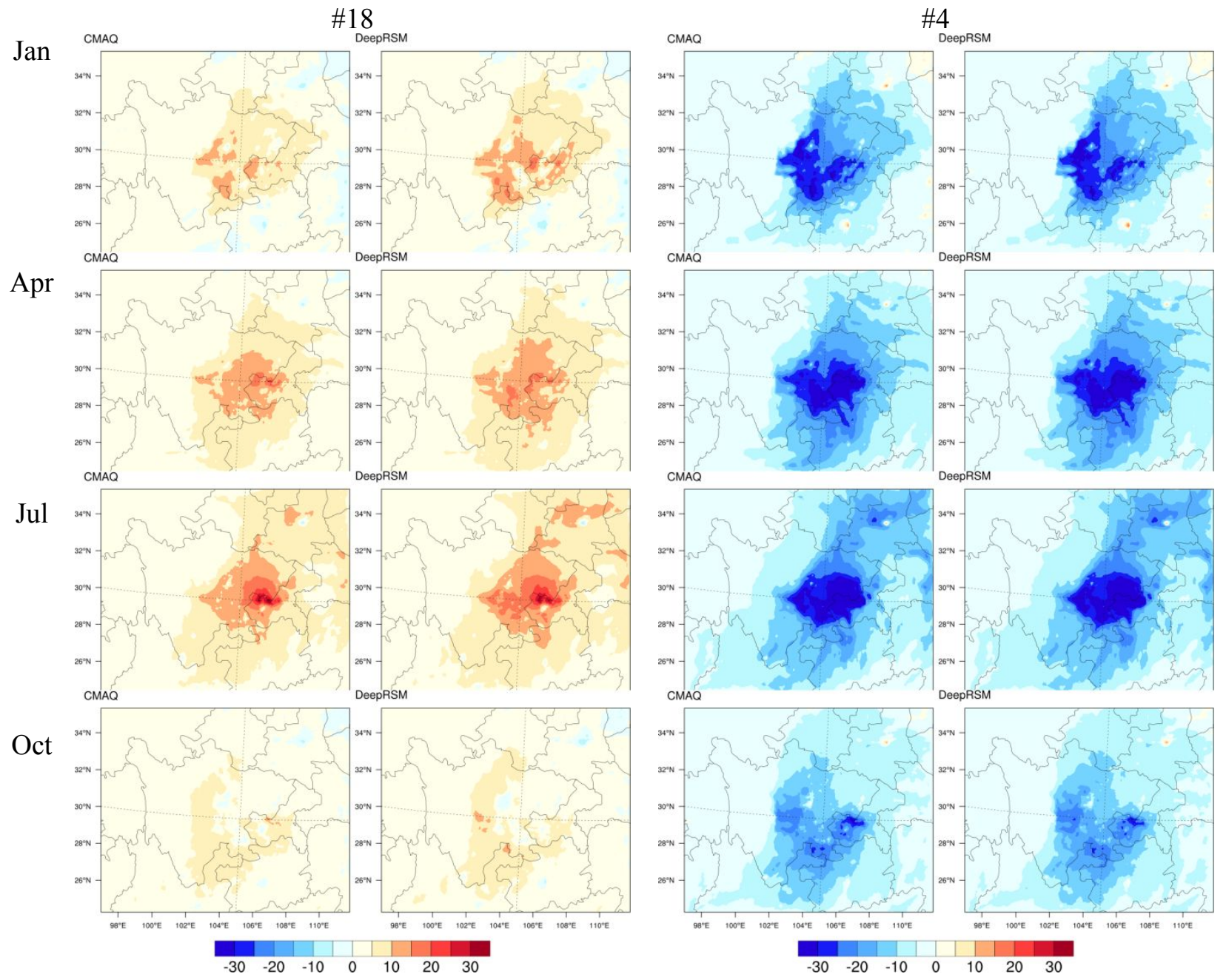

Figure S13 same as Figure S10 but for CYR 

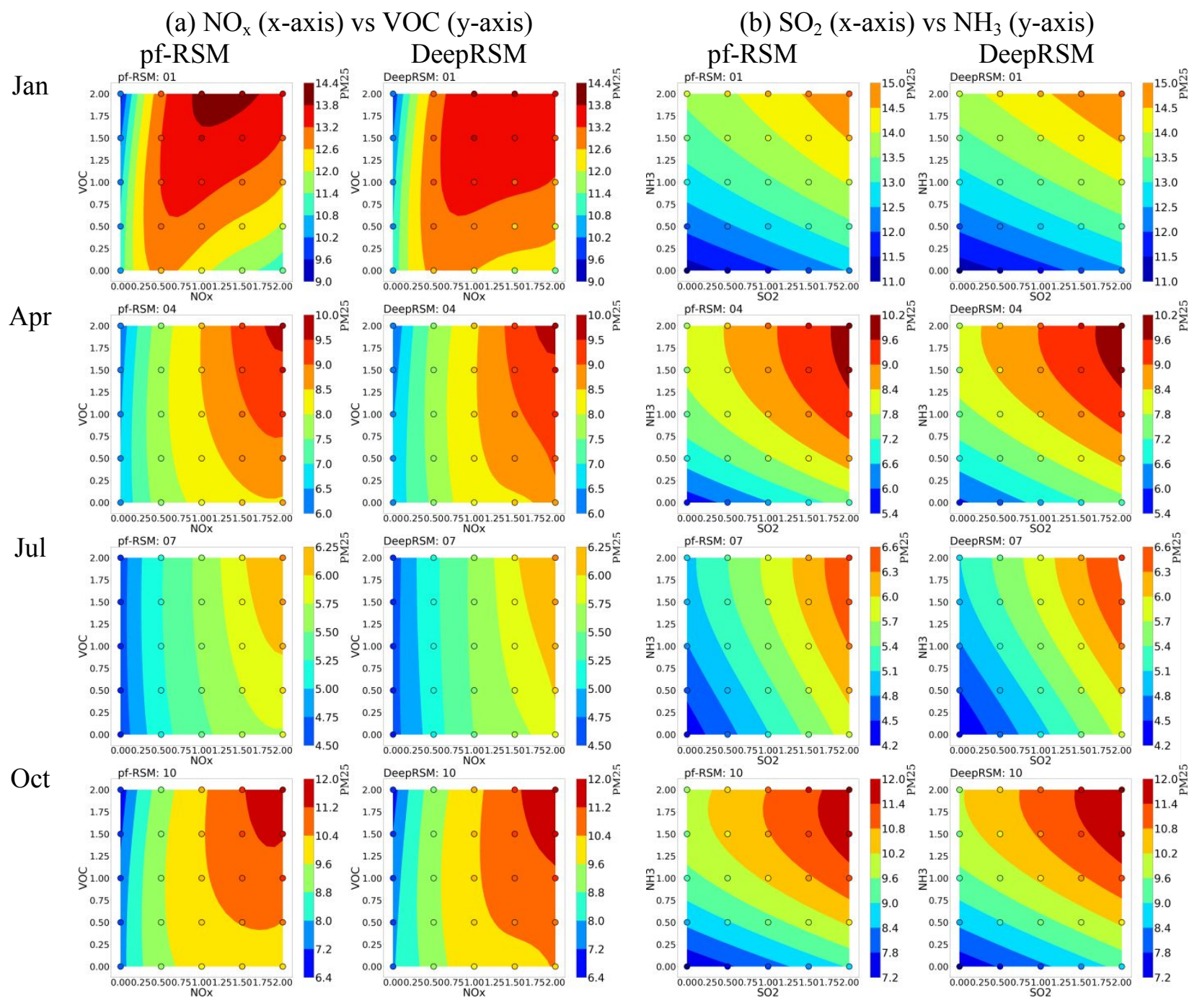

Figure S14. The isopleths of $\mathrm{PM}_{2.5}$ response to $\mathrm{NO}_{\mathrm{x}} / \mathrm{VOC}$ (a) and $\mathrm{SO}_{2} / \mathrm{NH}_{3}$ (b) emission change (baseline $=1$ ) predicted by pf-RSM and DeepRSM with -DT for CN27 domain (the values are averages of all grid cells and days; the color dots represent the simulated value in CMAQ) 

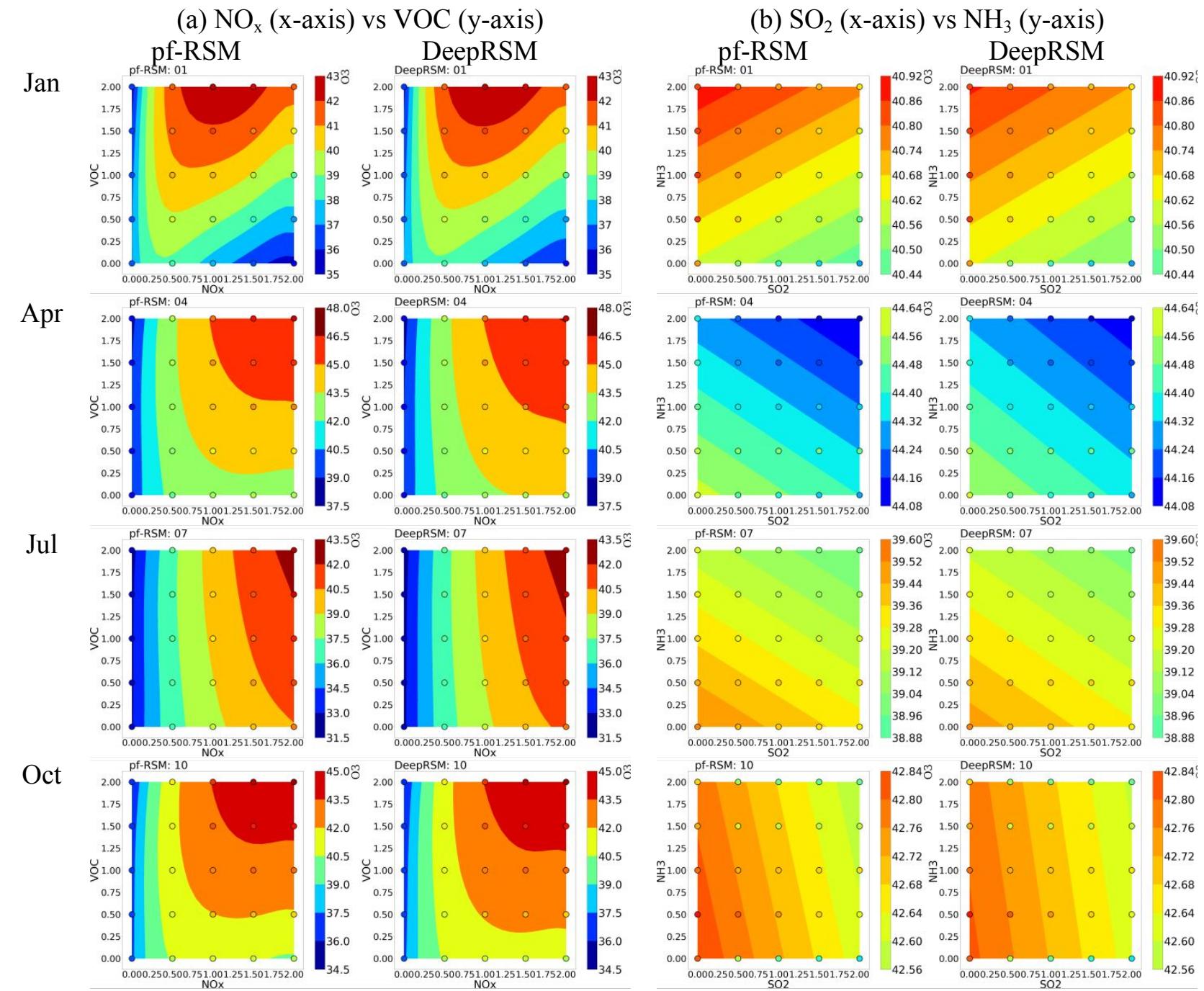

Figure $\mathrm{S} 15$. The isopleths of $\mathrm{O}_{3}$ response to $\mathrm{NO}_{\mathrm{x}} / \mathrm{VOC}$ (a) and $\mathrm{SO}_{2} / \mathrm{NH}_{3}$ (b) emission change (baseline $=$ 1) predicted by pf-RSM and DeepRSM with -DT for CN27 domain (the values are averages of all grid cells and days; the color dots represent the simulated value in CMAQ) 